Prospecção Tecnológica sobre a Utilização de Cacau Fino

\author{
Mendonça, T. A.; Dantas; E. A.; Gouvea, A. A. L.; Oliveira, R. S.; Alves, A. \\ R. C.; Padilha, F. F.; Machado, B. A. S.*
}

Rev. Virtual Quim., 2016, 8 (4), 1094-1103. Data de publicação na Web: 7 de agosto de 2016

http://rvq.sbq.org.br

\title{
Technology Assessment on the Use of Cocoa Fine
}

\begin{abstract}
The fine cocoa beans are a great international interest, for its particular characteristics of giving flavor to products. Then, the objective of this paper was to analyze the potential and technological developments, through patent applications in relation to cocoa. The survey was conducted from the combination of a keyword with the International Patent Code (IPC), the databases of the INPI patents and Espacenet. The most widely used CIP was the A23G1/00. It was observed a total of deposited documents between 1 and 4 per year, during the period 1912-2000. In 2001, was found a greater number of patent applications (9). The Great Britain stood out as the largest holder of patents relating to cocoa and cocoa products (40). It was noted that $98.39 \%$ of patent documents in Espacenet were directed mainly to the food industry sector and only $1.61 \%$ were intended for the pharmaceutical industry. The Brazil didn't have many deposited patents documents, so, there is a need for greater incentives for research and development (R\&D).
\end{abstract}

Keywords: Technological trends; Patents; Flavor.

\section{Resumo}

Os grãos de cacau fino são de grande interesse internacional por conferir características particulares de aroma e sabor aos produtos. Dessa forma, objetivou-se analisar as potencialidades e evolução tecnológica através dos depósitos de patentes em relação ao cacau. A pesquisa foi realizada a partir da combinação de uma palavra-chave com o Código Internacional de Patentes (CIP), nos bancos de dados de patentes do INPI e Espacenet. O CIP mais empregado foi o A23G1/00. Observou-se um total de documentos depositados entre 1 e 4 patentes por ano no período de 1912 a 2000. Em 2001, verificou-se um maior número de pedidos de patentes (9). A Grã-Bretanha destacou-se por ser o maior detentor das patentes referentes a cacau e produtos de cacau (40). Notou-se que $98,39 \%$ dos documentos de patentes no Espacenet estavam voltados principalmente para o setor da indústria de alimentos e apenas 1,61\% destinavam-se para a indústria farmacêutica. O Brasil não possuía muitos documentos de patentes depositados, dessa forma, existe a necessidade de maiores estímulos para Pesquisa e Desenvolvimento (P\&D).

Palavras-chave: Tendências tecnológicas; Patentes; Aroma/Sabor.

\footnotetext{
* Faculdade de Tecnologia SENAI CIMATEC, Serviço Nacional de Aprendizagem Industrial, Avenida Orlando Gomes, 1845, Piatã, CEP 41650-010, Salvador-BA, Brasil.

Mbrunamachado17@hotmail.com

DOI: $10.21577 / 1984-6835.20160078$
}

Rev. Virtual Quim. |Vol 8| |No.4| |1094-1103| 


\title{
Prospecção Tecnológica sobre a Utilização de Cacau Fino
}

\author{
Tácila A. Mendonça, ${ }^{a}$ Emanuelle A. Dantas, ${ }^{a}$ Ana Alice L. de Gouvea, ${ }^{a}$ \\ Roseane S. Oliveira, ${ }^{a}$ Aline R. C. Alves, ${ }^{a, b}$ Francine F. Padilha, ${ }^{b}$ Bruna \\ Aparecida S. Machado ${ }^{\mathrm{a} \cdot *}$ \\ ${ }^{a}$ Faculdade de Tecnologia SENAI CIMATEC, Serviço Nacional de Aprendizagem Industrial, \\ Avenida Orlando Gomes, 1845, Piatã, CEP 41650-010, Salvador-BA, Brasil.

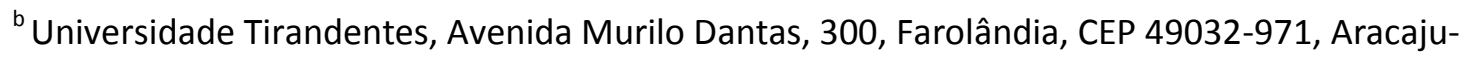 \\ $\mathrm{SE}$, Brasil. \\ * brunamachado17@hotmail.com
}

Recebido em 6 de agosto de 2016. Aceito para publicação em 6 de agosto de 2016

\section{Introdução \\ 2. Material e Métodos \\ 3. Resultados e Discussão \\ 4. Conclusões}

\section{Introdução}

O cacaueiro é uma planta nativa originária do continente Sul Americano, provavelmente das bacias dos rios Amazonas e Orinoco. ${ }^{1,2-4} \mathrm{O}$ interesse no seu cultivo está no aproveitamento das sementes para produção de derivados de cacau. ${ }^{5,6}$

Após processo artesanal de fermentação e posterior torrefação, as sementes são o principal produto comercializado, sendo utilizadas para a fabricação de produtos como: o chocolate, a manteiga de cacau, a torta e o pó. ${ }^{1,4,7-9 .}$

Com as variações geográficas e climáticas, as variedades de cacau inicialmente reconhecidas foram: Criollo, Forastero e Trinitário. ${ }^{10-11}$ Atualmente têm-se alguns outros grupos de cacau: Amelonado,
Contamana, Curaray, Guiana, Iquitos, Maranon, Nacional, Nanay e Purus. No mercado mundial os grãos de cacau se classificam em cacau ordinário e cacau fino ou de aroma. ${ }^{5,12}$ As variedades de cacau Crioulo e Trinitário são as mais utilizadas comercialmente por conferir características particulares de aroma e sabor em chocolates considerados de qualidade excelente, ou seja, chocolates finos. ${ }^{12}$ O flavor presente em produtos de cacau depende da variedade destes grãos, dos processos de fermentação e secagem e das condições de torrefação. ${ }^{13}$

Conforme o Acordo Internacional de Cacau, ${ }^{14}$ o cacau fino apresenta aroma e cor únicos, produzido, principalmente, por países da África (Jamaica, Madagascar, São Tomé e Príncipe), América Central (Granada, República Dominicana, Dominica, Santa Lúcia, Trindade e Tobago e Venezuela), América do Sul (Colômbia, Costa Rica, 
Equador e Peru), Ásia (Indonésia) e Oceania (Papua-Nova Guiné). O Brasil, apesar de ser o $5^{\circ}$ produtor mundial de cacau, tendo uma produção de 220 mil toneladas na safra $2011 / 12{ }^{15}$ não se encontra entre os produtores que exportam o cacau fino.

Por sua importância internacional devido às características sensoriais, principalmente o chocolate, verifica-se a necessidade de incentivar os produtores brasileiros para este tipo de cacau, agregando valor aos produtos e proporcionando o desenvolvimento de padrões de qualidade com atrativos comerciais e de interesse internacional, sendo possível estimar o desenvolvimento desta tecnologia e a extensão de sua aplicação através da Busca de Anterioridade. 16

Diante desse cenário, o objetivo deste trabalho foi elaborar um estudo de prospecção tecnológica para avaliar o panorama mundial de pesquisas desenvolvidas sobre o cacau e produtos de cacau fino, relacionando os documentos de patentes depositados sobre esta tecnologia a respeito de quais países são os principais depositantes, a evolução histórica dos depósitos e as principais áreas de aplicação.

\section{Material e Métodos}

Para a pesquisa da tecnologia protegida ou descrita em documentos de patentes, foi elaborada uma estratégia de busca que combinou os campos da Classificação Internacional de Patentes (CIP), associada a uma palavra-chave e a combinação das palavras-chave "cacau and aroma" e "cacau and sabor", na base de dados do European Patent Office (Espacenet) e do Instituto Nacional de Propriedade Industrial (INPI) do Brasil, respectivamente, visto que estas poderiam representar a forma como o cacau e produtos de cacau fino poderiam ser encontrados nos documentos de patentes.

Esta busca foi realizada entre os meses de abril a junho de 2014. Foi utilizada a pesquisa avançada (Advanced Search) e os campos de pesquisa "título" e "resumo" nos bancos de patentes durante o levantamento de dados. Foram excluídos documentos pertencentes à mesma família de patentes. A prospecção tecnológica foi realizada por meio de coleta, tratamento e análise das informações extraídas dos documentos de patentes encontrados.

\section{Resultados e Discussão}

Os resultados da combinação de dois critérios distintos, palavra-chave e/ou palavras-chave que poderiam descrever sobre o produto pesquisado e os códigos da CIP encontram-se na Tabela 1.

Constatou-se um universo de 1398 registros de documentos de patentes para a base de dados europeia e 37 nacional referentes à tecnologia de interesse. Todavia, estes números não representam o total de invenções protegidas nesta área, já que uma mesma patente pode ser depositada em diferentes países com o objetivo de garantir o direito de exclusividade aos seus inventores, uma vez que, o direito da patente é territorial.

Para a combinação da palavra-chave (Cocoa flavour*) com o CIP (A23G1) no Espacenet e as duas combinações de palavras-chaves, "cacau and aroma" e "cacau and sabor", no INPI por representarem o cacau e produtos de cacau fino, foram encontrados 124 documentos de patentes na base europeia e 31 na base do INPI.

A Figura 1 mostra o número de patentes por códigos e suas respectivas definições. 
Tabela 1. Busca de patentes por palavra-chave, agrupamento das palavras-chave e códigos da classificação internacional de patentes (CPI) na base de dados europeia (Espacenet) e nacional (INPI)

Palavra(s)-chave e/ou códigos

Cocoa flavour

Cocoa flavour*

Cocoa flavour and A23G1

Cocoa flavour* and A23G1

Cocoa flavour and A23G3

Cocoa flavour* and A23G3

Cocoa flavour and A23L1

Cocoa flavour* and A23L1

Cacau and aroma

\section{Número de Patentes Depositadas}

\section{Espacenet}

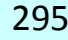

639

131

17

58

43

128

INPI

Cacau and sabor

A23G = cacau; produtos de cacau (exemplo: chocolate); substitutos de cacau ou produtos de cacau; confeitos; goma de mascar; sorvetes; preparações dos mesmo

A23L = alimentos, produtos alimentícios ou bebidas não alcoólicas, não abrangidos pelas subclasses A21D ou A23B-A23J; seu preparo ou tratamento (exemplo: cozimento, modificação das qualidades nutritivas, tratamento físico); conservação de alimentos ou produtos alimentícios, em geral

* agrupamento das palavras-chave

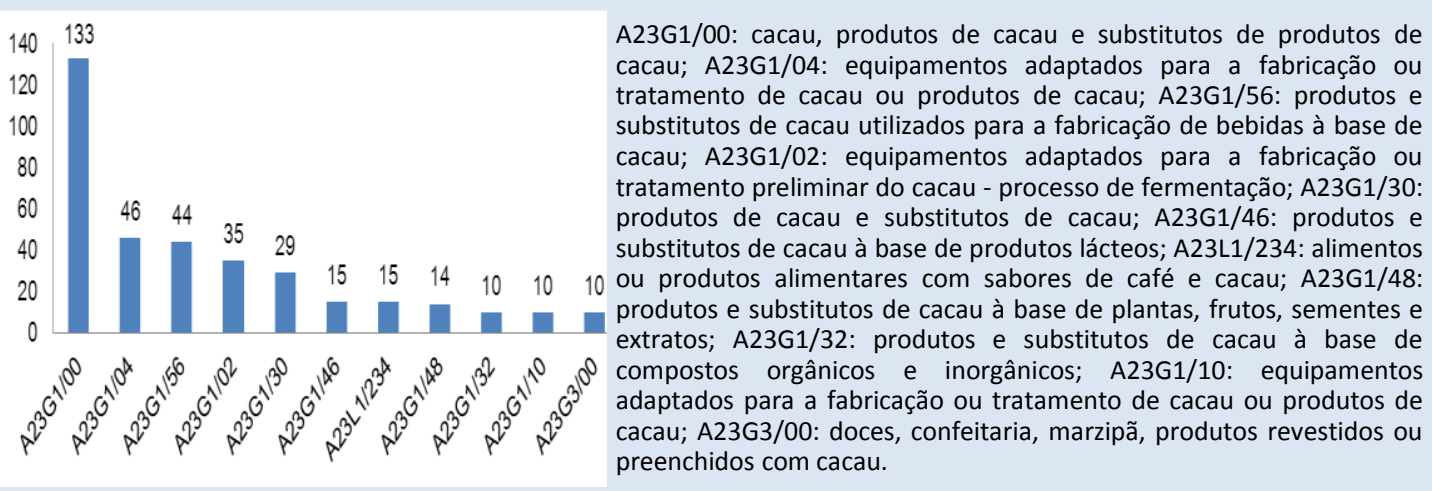

Figura 1. Distribuição das patentes relacionadas ao cacau e produtos de cacau fino por códigos da classificação internacional, na base de dados europeia Espacenet

Notou-se que a CIP mais empregada nos documentos de patentes foi o A23G1/00 (133) que se refere ao cacau, produtos de cacau e substitutos de produtos de cacau, seguido dos códigos A23G1/04 (46) que dizem respeito a equipamentos adaptados 
para a fabricação ou tratamento de cacau ou produtos de cacau (máquinas de torrefação, trituração ou moagem) e A23G1/56 (44) que trata de produtos e substitutos de cacau utilizados para a fabricação de bebidas a base de cacau.
A evolução histórica dos depósitos de patentes relacionados ao cacau entre os anos de 1912 a 2012 é demonstrada na Figura 2.

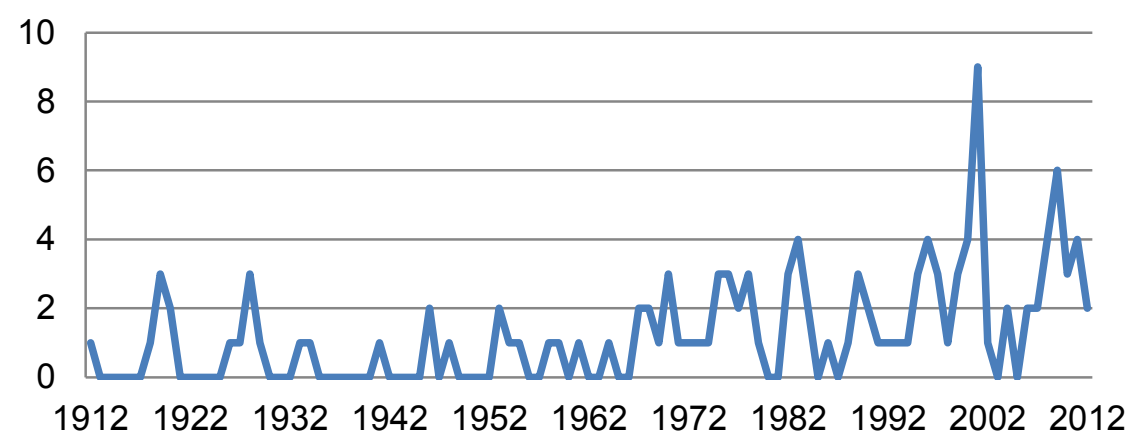

Figura 2. Evolução dos depósitos de patentes sobre o cacau e produtos de cacau fino entre 1912 e 2012, verificada na base de dados europeia Espacenet.

A primeira patente identificada a respeito da tecnologia de interesse ocorreu em 1912 na Grã-Bretanha. Estimou-se entre 1912 a 2000 um total de 1 e 4 patentes por ano. Todavia, em 2001, houve maior presença de pedidos de patentes (9).

Ao analisar os documentos no INPI, foi verificado que os principais detentores de pedidos eram empresas privadas nacionais e multinacionais, com 16 documentos de patentes, seguidos de 12 depósitos de pessoa física e 2 de universidades estaduais (Universidade Estadual de Feira de Santana UEFS/BA e Universidade Estadual de Campinas - Unicamp/SP).
Nota-se que as patentes brasileiras depositadas no INPI (Figura 3), apresentavam como principais detentores da titularidade o Paraná $(23,33 \%)$ e São Paulo (16,67\%), assim como o Reino Unido (20,00\%) e Estados Unidos (13,33\%). Isto ratifica o fato de que uma patente pode ser depositada em diferentes países com o objetivo de garantir o direito de exclusividade aos depositantes nos mercados considerados mais interessantes e relevantes para se realizar um investimento comercial e proporcionar fins lucrativos para o país financiador. ${ }^{15,17-18}$

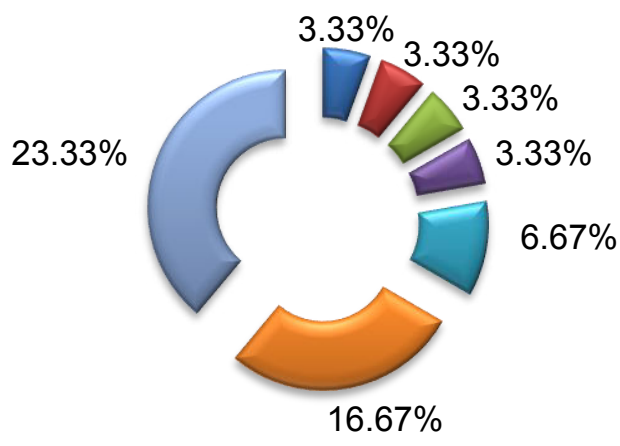

Bahia

- Espírito Santo

$\square$ Mato Grosso

$\square$ Rio de Janeiro

$\square$ Santa Catatina

$\checkmark$ São Paulo

๑Paraná 


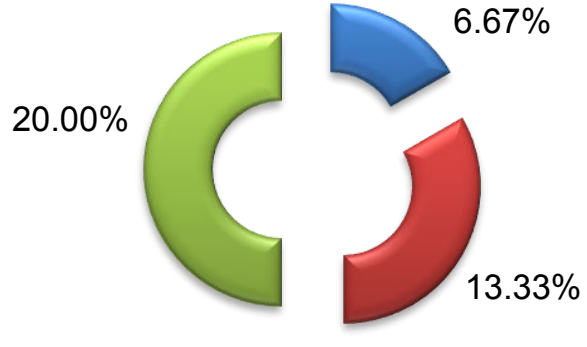

\author{
- Espacenet \\ - Estados Unidos \\ $\square$ Reino Unido
}

Figura 3. Principais estados brasileiros e países detentores de pedidos de patentes no INPI

É importante salientar que estes resultados podem estar associados a um maior investimento financeiro em Ciência e Tecnologia (C\&T) pelos países investidores, com o intuito de proporcionar maiores avanços na área de pesquisa e de desenvolvimento da tecnologia de interesse. Esta ação traz impactos positivos, visto que pode induzir ao desenvolvimento de novos processos tecnológicos e impulsionar áreas de P\&D. ${ }^{15,17-18}$

Apesar destes investimentos, o Brasil ainda não consolidou seu sistema de inovação, devido à falta de cultura local a respeito do tema e de articulação eficiente entre governo, empresas e universidades capazes de promover um sistema de P\&D eficaz. Este cenário, por sua vez, proporciona um menor número de patentes depositadas.

$\mathrm{Na}$ Tabela 2 encontram-se uma síntese a respeito dos principais estudos e compostos analisados tendo como matéria-prima o cacau, entre os anos de 2004 a 2013.

Os estudos voltavam-se principalmente para análise de compostos fenólicos, devido a reconhecida atividade antioxidante. Nos últimos anos houve um considerado aumento no número de estudos sobre os polifenóis presentes no cacau. Este fato deve-se principalmente aos benefícios que desempenham sobre a saúde humana, como prevenção de reações oxidativas e de formação de radicais livres, proteção contra danos ao DNA das células ${ }^{25}$, propriedades anti-inflamatórias, antiaterogênica,

anticarcinogênica, antitrombótica, antimicrobiana, analgésica e vasodilatadora, o que Ihes conferem um elevado potencial farmacológico. ${ }^{26}$

Os polifenóis são componentes importantes do sabor do cacau, sendo responsáveis pelo amargor e adstringência. Além disso, os produtos da condensação de compostos fenólicos gerados durante 0 processo de fermentação e secagem conferem a cor marrom do cacau e do chocolate. $^{27}$

A partir da análise dos resultados dos documentos de patentes, no que diz respeito aos países nos quais se originou a tecnologia patenteada, foi possível observar que esta tecnologia se encontra bastante centralizada nos países mais desenvolvidos. Como demonstrado na Figura 4, a Grã-Bretanha (40) destacou-se por ser o maior detentor das patentes referentes a cacau e produtos de cacau, seguido da Alemanha (18), Escritório Europeu de Patentes (15) e Estados Unidos (14). Nota-se que o Brasil apresentou apenas um documento de patente depositado sobre o tema na base de dados europeia.

Verificou-se que $98,39 \%$ dos documentos de patentes encontrados no Espacenet estavam voltados principalmente para 0 setor da indústria de alimentos e apenas $1,61 \%$ destinavam-se para a indústria farmacêutica. 
Tabela 2. Identificação dos principais estudos e compostos analisados com o cacau entre os anos de 2004 a 2013

\begin{tabular}{|c|c|c|c|}
\hline Amostra & Local de origem & Composto analisado & Autor \\
\hline $\begin{array}{c}\text { Theobroma cacao L. } \\
\text { (Variedades: Criollo, } \\
\text { Trinitário, Nacional e } \\
\text { Forastero) }\end{array}$ & $\begin{array}{l}\text { África (Costa do Marfim, } \\
\text { Gana e Madagascar), } \\
\text { América (Equador e } \\
\text { Trindade) e Ásia (Java e } \\
\text { Nova Guiné) }\end{array}$ & $\begin{array}{l}\text { Compostos fenólicos } \\
\text { (procianidinas) e flavor }\end{array}$ & $\begin{array}{l}\text { COUNET et } \\
\text { al. }(2004)^{13}\end{array}$ \\
\hline $\begin{array}{l}\text { Theobroma cacao L. } \\
\text { (Genótipos: IAC1, } \\
\text { ICS95, IMC67, P7, } \\
\text { SCA12, UF29, UF61, } \\
\text { UF667 e UF668) }\end{array}$ & América (Brasil) & $\begin{array}{l}\text { Compostos fenólicos } \\
\text { (protocianidinas, } \\
\text { flavan-3-óis e } \\
\text { procianidinas) }\end{array}$ & $\begin{array}{l}\text { EFRAIM et } \\
\text { al. }(2006)^{19}\end{array}$ \\
\hline $\begin{array}{c}\text { Theobroma cacao L. } \\
\text { (Variedades: } \\
\text { Forastero, } \\
\text { Amazônico, Forastero } \\
\text { Amazônico, Hibrido } \\
\text { Amazônico, Trinitário, } \\
\text { Criollo e } \\
\text { Desconhecido) }\end{array}$ & $\begin{array}{l}\text { África (Costa do Marfim e } \\
\text { Camarões), América } \\
\text { (Colômbia, Equador, Guiné } \\
\text { Equatorial, Venezuela, Peru } \\
\text { e, República Dominicana) e } \\
\quad \text { Ásia (Malásia) }\end{array}$ & $\begin{array}{l}\text { Compostos fenólicos } \\
\text { (polifenóis) e } \\
\text { atividades } \\
\text { antioxidantes pelo } \\
\text { método DPPH, ABTS, } \\
\text { FRAP,ORAC. }\end{array}$ & $\begin{array}{l}\text { HII et al. } \\
(2009)^{20}\end{array}$ \\
\hline $\begin{array}{c}\text { Theobroma cacao } \\
\text { L.(Variedade: } \\
\text { Forastero) }\end{array}$ & América (Brasil) & $\begin{array}{l}\text { Compostos fenólicos } \\
\text { (polifenóis totais, } \\
\text { flavan-3-óis e } \\
\text { procianidinas) e } \\
\text { aceitação sensorial }\end{array}$ & $\begin{array}{l}\text { EFRAIM et } \\
\text { al. }(2010)^{21}\end{array}$ \\
\hline $\begin{array}{c}\text { Theobroma cacao L. } \\
\text { (Clones da variedade } \\
\text { Criollo) } \\
\text { Theobroma cacao L. } \\
\text { (cultivares resistentes }\end{array}$ & América (Venezuela) & $\begin{array}{c}\text { Compostos fenólicos } \\
\text { totais }\end{array}$ & $\begin{array}{l}\text { ELWERS et. } \\
\text { al. }(2010)^{2}\end{array}$ \\
\hline $\begin{array}{l}\text { - SR162 e PH16 e não } \\
\text { resistentes - Pará, } \\
\text { Parazinho e } \\
\text { Maranhão da } \\
\text { variedade Forastero) }\end{array}$ & América (Brasil) & $\begin{array}{c}\text { Compostos fenólicos e } \\
\text { metilxantinas }\end{array}$ & $\begin{array}{l}\text { LEITE et al. } \\
(2013)^{22}\end{array}$ \\
\hline $\begin{array}{l}\text { Theobroma cacao L. } \\
\text { (Genótipos: SCA6, } \\
\text { EET62 e CCN51) }\end{array}$ & América (Costa Rica) & Compostos voláteis & $\begin{array}{l}\text { KADOW et } \\
\text { al. }(2013)^{23}\end{array}$ \\
\hline $\begin{array}{c}\text { Theobroma cacao } \mathrm{L} \text {. } \\
\text { (variedade: } \\
\text { Forastero) }\end{array}$ & América (Brasil) & $\begin{array}{c}\text { Qualidade } \\
\text { microbiológica }\end{array}$ & $\begin{array}{l}\text { MELO NETO } \\
\text { et al. } \\
(2013)^{24}\end{array}$ \\
\hline
\end{tabular}




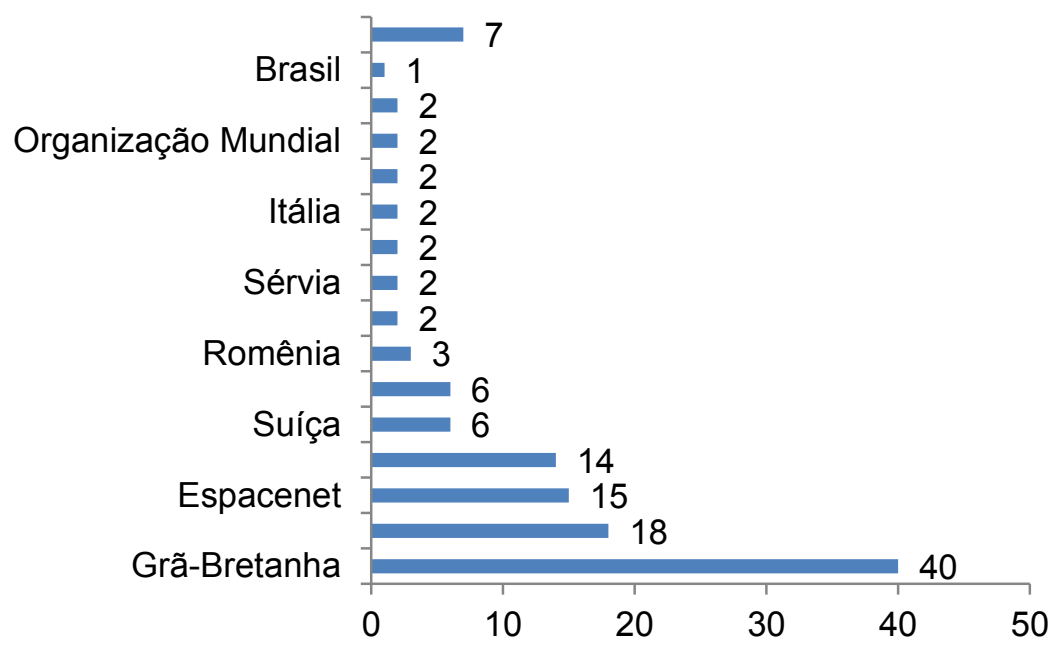

Figura 4. Distribuição de depósitos de patentes relacionados ao cacau e produtos de cacau fino, por país de origem dos depositantes, na base de dados europeia (Espacenet)

Observou-se que os documentos de patentes com aplicações na indústria de alimentos destinavam-se para elaboração de produtos de cacau $(62,10 \%)$; métodos de processamento de cacau $(10,48 \%)$ que compreendem procedimentos para agregar sabor e aroma das sementes, elaboração de novos equipamentos para aprimorar processos de fermentação, secagem, torrefação, moagem e manufatura das sementes; elaboração de produtos substitutos de cacau $(9,67 \%)$; processo de aromatização de alimentos (9,67\%), biotecnologia (6,45\%) que abrangia a utilização de peptídeos e enzimas como precursores de aroma e desenvolvimento de embalagens; e medicamentos $(1,60 \%)$.

Os documentos de patentes depositados no INPI estavam voltados principalmente para a indústria de alimentos $(80,00 \%)$, assim como verificados na base de dados do Espacenet. As demais áreas de aplicação foram biotecnologia $(16,67 \%)$ e indústria farmacêutica (3,33\%).

\section{Conclusões}

O maior número de patentes depositadas referentes à cacau e produtos de cacau, encontrava-se em domínio da Grã-Bretanha, sendo mais depositadas na Espacenet, no setor da indústria de alimentos em que o foco foi a elaboração de produtos de cacau, havendo assim a necessidade de difundir informações e incentivar novos investimentos para melhorar o panorama nacional em relação a $C \& T$ e $P \& D$, além da utilização de políticas governamentais e estratégias empresariais que possibilitem impulsionar a realização de atividades voltadas para a geração de novas tecnologias.

\section{Agradecimentos}

Os autores agradecem a Fundação de Amparo à Pesquisa do Estado da Bahia (FAPESB) pelo financiamento concedido ao projeto "Identificação e caracterização de cacau fino por painel sensorial e sua correlação com o perfil de aromas voláteis obtidos por cromatografia gasosa acoplada a espectrometria de massas" - DTE0029/2013. 


\section{Referências Bibliográficas}

${ }^{1}$ SUFRAMA - Superintendência da Zona Franca de Manaus. Cacau. Fundação Getúlio Vargas, Instituto Superior de Administração e Economia, Zona Franca de Manaus: projeto potencialidades - estudo de viabilidade econômica, 2003, 3. [Link]

${ }^{2}$ Elwers, S.; Zambrano, A.; Rohsius, C.; Lieberei, R. Histological features of phenolic compounds in fine and bulk cocoa seed (Theobroma cacao L.). Journal of Applied Botany and Food Quality 2010, 83, 182. [Link]

${ }^{3}$ Guimarães, M. M.; Figueiredo, T. V. B.; Machado, B. A. S.; Druzian, J. Utilização de chocolates ricos em polifenóis e com ação antioxidante: busca em bases de patentes. Cadernos de Prospecção 2012, 5, 168. [CrossRef]

${ }^{4}$ Gonzales, A. D. F.; Vital, A. V. D.; Lima, J. M.; Rodrigues, M. B. S. Desenvolvimento sustentável para o resgate da cultura do cacau baseado no aproveitamento de resíduos. Interfaces Científicas - Saúde e Ambiente 2013, 1, 41. [Link]

${ }^{5}$ Cruz, J. F. M. Caracterização das sementes de variedades de cacau Theobroma cacau L. resistentes à vassoura de bruxa durante a fermentação e após a secagem. 2012. 101 f. Dissertação (Mestrado em Ciência de Alimentos), Programa de Pós-graduação em Ciência de Alimentos, Faculdade de Farmácia, Universidade Federal da Bahia, Salvador, 2012. [Link]

${ }^{6}$ Alves, A. B.; Bragagnolo, N. Determinação simultânea de teobromina, teofilina e cafeína em chás por cromatografia líquida de alta eficiência. Revista Brasileira de Ciências Farmacêuticas 2002, 38, 237. [CrossRef]

${ }^{7}$ Gramacho, I. C. P.; Magno, A. E. S.; Mandarino, E. P.; Matos, A. Cultivo e beneficiamento do cacau na Bahia. Ilhéus, CEPLAC. 1992, 124.

${ }^{8}$ Leite, J. B. V. Cacaueiro: propagação por estacas caulinares e plantio no semi-árido do estado da Bahia. 2006. 75 f. Tese (Doutorado em Agronomia), Faculdade de Ciências Agrárias e Veterinárias, Universidade Estadual Paulista, Jaboticabal, 2006. [Link]
${ }^{9}$ Oetterer, M.; Regitano-D'arce, M. A. B.; Spoto, M. H. F. Fundamentos de ciência e tecnologia de alimentos. Barueri, SP: Manole, 2006, 612.

${ }^{10}$ Batista, A. P. S. A. Chocolate: sua história e principais características. 2008. $56 \mathrm{f}$. Monografia (Especialização em Gastronomia e Saúde), Programa de Pós-graduação Lato Sensu, Centro de Excelência Turismo, Brasília, 2008. [Link]

${ }^{11}$ Sena, J. V. C. Produção e efetivo do cacau no nordeste. Informe Rural Etene, Escritório Técnico de Estudos Econômicos do Nordeste, 2011, 2, ano V. [Link]

${ }^{12}$ Beckett, S. T. Industrial chocolate manufacture and use. 4a. ed., Chapman and Hall: London, 2009, 20-23.

${ }^{13}$ Counet, C.; Ouwerx, C.; Rosoux, D.; Collin, $\mathrm{S}$. Relationship between procyanidin and flavor contents of cocoa liquors from different origins. Journal of Agricultural and Food Chemistry 2004, 52, 6243. [CrossRef] [PubMed]

${ }^{14}$ United Nations, United Nations Conference on Trade and Development (UNCTAD). International Cocoa Agreement (2010), Geneva, 2011. Disponível em: http://www.icco.org/about-us/internationalcocoa-agreements/cat_view/2-icco-

agreements-and-their-history/3-2010-

international-cocoa-agreement.html. Acesso em: 15 maio 2014.

${ }^{15}$ ICCO (International Cocoa Organization), Production of cocoa beans (2014). Disponível em: http://www.icco.org/aboutus/international-cocoa-

agreements/cat_view/30-related-

documents/46-statistics-production.html.

Acesso em: 16 maio 2014.

${ }^{16}$ Quintella, C. M.; Meira, M.; Guimarães, A. K.; Tanajura, A. S.; da Silva, H. R. G. Prospecção tecnológica como uma ferramenta aplicada em ciência e tecnologia para se chegar à inovação. Revista Virtual de Química 2011, 3, 406. [CrossRef]

${ }^{17}$ Mendonça, T. A.; Druzian, J. I.; Nunes, I. L. Prospecção tecnológica da utilização da Spirulina platensis. Cadernos de Prospeç̧ão Tecnológica 2012, 5, 44. [CrossRef]

${ }^{18}$ Mendonça, T. A.; Cazumbá, Í; Lima, A. B.; Nunes, I. L. Prospecção tecnológica da 
utilização de microalgas em processo de extração de carotenoides voltados para insumos na nutrição humana e animal. Revista GEINTEC 2013, 3, 193. [CrossRef]

${ }^{19}$ Efraim, P.; Tucci, M. L.; Pezoa-García, N. H.; Haddad, R.; Eberlin, M. N. Teores de compostos fenólicos de sementes de cacaueiro de diferentes genótipos. Brazilian Journal Food Technology 2006, 9, 229. [Link]

${ }^{20}$ Hii, C. L.; Law, C. L.; Suzannah, S.; Misnawi; Cloke, M. Polyphenols in cocoa (Theobroma cacao L.). Asian Journal of Food and AgroIndustry 2009, 2, 702. [Link]

${ }^{21}$ Efraim, P.; Pezoa-García, N. H.; Jardim, D. C. P.; Nishikawa, A.; Haddad, R.; Eberlin, M. N. Influência da fermentação e secagem de amêndoas de cacau no teor de compostos fenólicos e na aceitação sensorial. Ciência $e$ Tecnologia de Alimentos 2010, 30, 142. [CrossRef]

${ }^{22}$ Leite, P. B.; Maciel, L. F.; Opretzka, L. C. F.; Soares, S. E.; Bispo, E. S. Phenolic compounds, methylxanthines and antioxidant Activity in cocoa mass and chocolates produced from "witch broom disease" resistant and non resistant cocoa cultivars. Ciência e Agrotecnologia 2013, 37, 244. [CrossRef]

${ }^{23}$ Kadow, D.; Bohlmann, J.; Phillips, W.; Lieberei, R. Identification of main fine or flavour components in two genotypes of the cocoa tree (Theobroma cacao L.). Journal of Applied Botany and Food Quality 2013, 86, 90. [CrossRef]

${ }^{24}$ Melo Neto, B. A.; Carvalho, E. A.; Netto Mello, D. L.; Anjos, A. C.; Ferreira, A. C. R.; Sacramento, C. K. Classificação de amêndoas e qualidade de líquor de cacau (Theobroma cacao L.) superior Bahia. Revista Brasileira de Produtos Agroindustriais 2013, 15, 391. [Link] ${ }^{25}$ Efraim, P.; Alves, A. B.; Jardim, D. C. P. Polifenóis em cacau e derivados: teores, fatores de variação e efeitos na saúde. Brazilian Journal Food Technology 2011, 14, 181. [CrossRef]

${ }^{26}$ Coutinho, M. A. S.; Muzitano, M. F.; Costa, S. S. Flavonoides: potenciais agentes terapêuticos para o processo inflamatório. Revista Virtual de Química 2009, 1, 241. [CrossRef]

${ }^{27}$ Leite, P. B.; Dissertação Mestrado, Universidade Federal da Bahia, Salvador, 2012. [Link] 